\title{
Journal of Biomedical and Allied Research
}

\section{Unilateral Hypertrophy of the Inner Lip}

\author{
Mario Cutrone ${ }^{1}$, Enrico Valerio ${ }^{2}$ and Ramon Grimalt ${ }^{3 *}$ \\ ${ }^{1}$ Unità Operativa di Pediatria, Ospedale Dell'angelo, Venice, Italy \\ ${ }^{2}$ Neonatal Intensive Care Unit, Ospedale San Bortolo, Vicenza, Italy
}

${ }^{3}$ Facultat de Medicina i Ciències de la Salut. UIC-Barcelona, Universitat Internacional de Catalunya, Sant Cugat del Vallès, Barcelona, Spain

"Corresponding Author: Ramon Grimalt, Facultat de Medicina i Ciències de la Salut, Universitat Internacional de Catalunya, Barcelona, Spain. Tel +34617301661.

Received Date: 09-09-2019; Accepted Date: 09-23-2019; Published Date: 09-30-2019

Copyright $^{\odot} 2019$ by Cutrone $\mathrm{M}$, et al. All rights reserved. This is an open access article distributed under the terms of the Creative Commons Attribution License, which permits unrestricted use, distribution, and

reproduction in any medium, provided the original author and source are credited.

\begin{abstract}
Variations on normality on the vulvar aspect have generated many difficulties among general pediatricians. The great amount of aesthetical perfection has led to an increasing number of adolescents seeking for vulvar correction. Here we describe a common alteration and provide some clues on the eventual surgical managing of the condition.
\end{abstract}

\section{Keywords}

Inner lips; Hypertrophy; Labioplasty; Labiothomy; Vulva

\section{Introduction}

The inner lips are two thin folds of the squamous layered epithelium, non-keratinized and free of hair. Sebaceous and eccrine glands, which open directly to the surface, are present in the connective sub epithelial tissue. Even though there is no fat, there is abundant vascularization, nerve endings and sensory receptors that provide them with great sensibility. Their anatomical features had already been largely described, but it was not until 1899 that Waldeyer collected measurements and established a normal size rank between 2.5 and $3.5 \mathrm{~cm}$. The most recent studies identify a variation of up to $2 \mathrm{~cm}$, with a range between 0.7 and $5 \mathrm{~cm}$, a medium length of $20-30 \mathrm{~mm}$, a width of $15 \mathrm{~mm}$ and a thickness of $4 \mathrm{~mm}$ [1]. The function of the lips is to prevent vaginal dryness and to guide the urinary flow. For this purpose it is necessary for them to measure at least $1 \mathrm{~cm}$. The quest for aesthetic perfection now includes genitalia. New aesthetic procedures such as vulvar and anal bleaching, tattoos and piercings 
amongst others involve the genital area. We see an increasing number of patients asking for advice about the technical options to correct this minimal defect that can be psychologically embarrassing.

\section{Clinical case}

A 3 year old girl was examined at our consultancy for abnormally enlarged genitalia and a unilateral inner labia enlargement was evidenced. The size of the right labia was $3.8 \mathrm{~cm}$ while the left labia were $1.6 \mathrm{~cm}$ (Figure 1). We reassured the mother about the benignity of the condition and explained to her how it could be surgically corrected after puberty.

Figure1: Unilateral Unilateral hypertrophy of the inner lip.

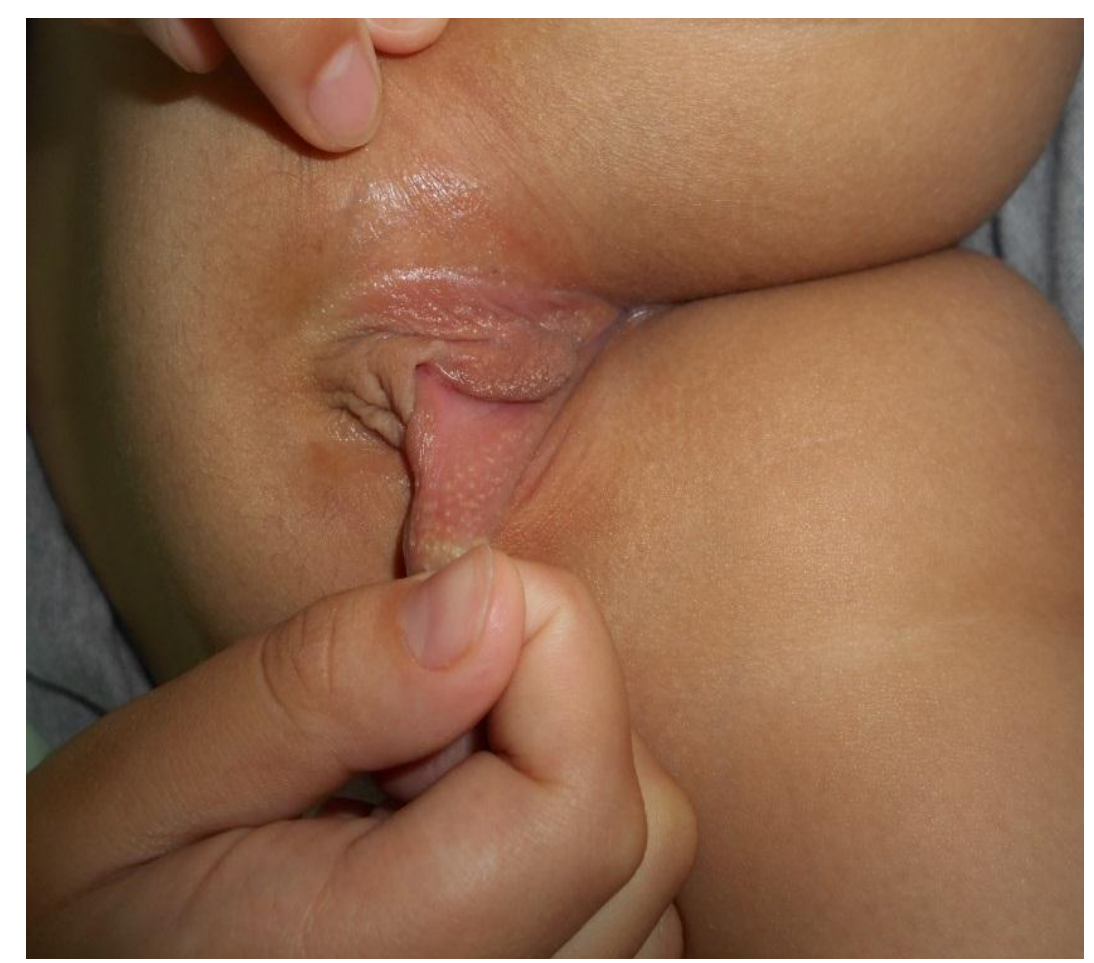

\section{Discussion}

Inner labia hypertrophy was defined in 1984 by Hodgkinson and Hait, even though descriptions of circumcision of the inner lips and clitoris had already been vaguely mentioned in previous texts [2]. Natural variations in the size of the inner lips in women are common. Size is independent of age, race, parity, hormonal use, etc. It is defined as the lip tissue that bulges further that the inner lips. Many authors consider hypertrophy when the size is more than 4-5cm [3]. The main cause for hypertrophy is idiopathic. Non-idiopathic forms have also been described as in cases of myelodysplasia, lymphedema, chronic irritation, inflammation (Crohn, endometriosis) by the action of exogenous androgenic hormones and tissues' laxity as a result of ageing [4]. Manipulation or forced mechanical stimulation has been observed in 
some African cultures (apron of Hotentotes women). Scientific evidence exists proving that the inflammation is linked to the growth of the inner lips and that there is an obvious relationship between the inflammation and the amount of estrogens circulating and the growth of the tissue. Spikes of circulating estrogens or episodes of punctual inflammation can explain the high variability of the size of the inner lips. The vast majority of hypertrophy cases take place at the beginning of puberty and, usually, not in a symmetric form. It can get worse during pregnancy and in advanced age. In the case of young women, we have to consider that the growth of the outer lips takes place later than the one of the inner lips, and in many occasions they end up covering them, not being defined as hypertrophy anymore or becoming bilateral. The hair of the vulva can also help mask enlarged lips. Largely, hypertrophy of the inner lips is asymptomatic and is not considered a malformation nor a disease. Nevertheless, in some cases, it can cause functional, aesthetic and psychological problems. The most frequent mentioned annoyances are pain, irritation during exercise or during sexual intercourse and difficulties with personal hygiene. Many techniques of reduction or labioplasty are available and largely described in literature. Complications are minimal, and the post-operatory sexual function and a woman's long-term satisfaction are usually excellent. Even if, as in our case, we receive consultation in infanthood, In general, it is advisable to delay the surgery, until adulthood. In recent years, cosmetic genital surgery has become popular, possibly as a result in the rise in public exposure of the naked female form on social media and the much 'sought after' waxed or shaven vulva. In addition, the use of tight clothing increases the desire for non-vulvar protuberance. The tendency for body comparison between young girls, sometimes coupled with their partner's opinion cab result in a loss of self-esteem, shame and anxiety. The vast majority of the publications suggest that consultations are mostly because of psychological discomfort. In some occasions we see patients who ask for a labial reduction but had a lip size less than $2-3 \mathrm{~cm}$, very far from what is considered hypertrophy. The dysmorphophobia of these patients is guided by distorted and manipulated beauty criteria. Some authors consider that genitoplasy differs only slightly from female genital mutilation. WHO defines it as the resection of any part of the external female genitalia for non-medical reasons?

\section{Conclusion}

In healthy women, there is wide variation in the size of these structures. Hypertrophy, uni or bilateral, is a consequence of the response of the tissue to a transitory stimulus that takes place with a higher frequency at the beginning of puberty. Even though different classifications exist, there is no international consensus for its diagnosis. Generally, it does not cause its recipient physical dysfunction or alteration. We need to reassure young women and their parents, as well as educate them in terms of the variations of the normality, meticulous hygiene and the use of looser cotton clothing for sports, to prevent them from a dysmorphic image of their body. Labioplasty should only be offered to patients with a real hypertrophy of the inner lips and a functional and/or psychological impact, and always advising that such surgery should be undergone during adulthood. 


\section{References}

1. Clerico C, Lari A, Mojallal A, Boucher F. Anatomy and aesthetics of the labia minora: the ideal vulva? Aesthetic Plas Surg. 2017;41(3):714-9.

2. Munhoz AM, Filassi JR, Ricci MD. Aesthetic labia minora reduction with inferior wedge resection and superior pedicle flap reconstruction. Plast Reconstr Surg 2015;35(4):419-31.

3. Kato K, Kondo A, Gotoh M, Tanaka J, Saitoh M, Namiki Y. Hypertrophy of labia minora in myelodysplastic women: Labioplasty to ease clean intermittent catheterization. Urology. 1988;31(4):294-6.

4. Bragagnini RP, Alvarez GN, Gonzalez RY, Ruiz DT, Escart in VR, Gonzalez MP. (2016) Hypertrophy of labiaminora: A growing problem in adolescence. Cir Pediatr. 2016;28(2):196. 